\title{
Productos de tabaco calentado con especial referencia a IQOS
}

\author{
SERGIO BELLO S.*
}

\section{Heated tobacco products with special reference to IQOS}

Heated Tobacco Products (HTP) are new tobacco consumption devices that are presented as a harm reduction product. The most widespread is IQOS by Philip Morris. In the IQOS aerosol, toxic substances are detected in a smaller amount and concentration than those detected in conventional cigarettes, with the exception of some of them. These substances are able of inducing disease. They could modify bronchial epithelial cells and vascular endothelium and could cause additional damages, such as hepatotoxicity. The amount of nicotine in IQOS is very similar to conventional cigarettes, so it is as addictive as a normal cigarette. The concentration of toxic substances emitted to the environment is lower than those of conventional cigarettes, but there is a health's risk of exposed non-smokers. Most people use HTP as a complement to conventional cigarettes, not as an alternative, becoming dual smokers. IQOS can create new generations addicted to nicotine, in addition to renormalize the tobacco's use in society. Many Medical Societies of Respiratory Diseases around the world have manifested against the use of HTP, and have proposed that they should be subject to the same regulatory policies that applied to all tobacco products, in line with the WHO Framework Convention on Tobacco Control.

Key words: Tobacco; Nicotine; Smokers; tobacco products; behavior addictive; Chemical and drug induced liver injury.

\section{Resumen}

Los Productos de Tabaco Calentado (PTC) son nuevos dispositivos de consumo de tabaco que se presentan como un producto de reducción del daño. El más difundido es IQOS de Philip Morris. En el aerosol de IQOS se detectan sustancias tóxicas en menor cantidad y concentración que las detectadas en el humo del cigarrillo convencional, a excepción de algunas. Estas sustancias son capaces de producir enfermedad, con alteración de las células del epitelio bronquial y del endotelio vascular y podría producir nuevos daños, como hepato-toxicidad. La cantidad de nicotina de IQOS es muy similar a los cigarrillos convencionales, por lo que es tan adictivo como el cigarrillo normal. La concentración de sustancias tóxicas emitidas al medio ambiente es menor que las del cigarrillo convencional, pero hay riesgo para la salud de los no fumadores expuestos. La mayoría de las personas usan los PTC como complemento a los cigarrillos convencionales, no como alternativa, transformándose en fumadores duales. IQOS puede crear nuevas generaciones adictas a la nicotina, además de renormalizar el consumo de tabaco en la sociedad. Muchas Sociedades Médicas de Enfermedades Respiratorias en el mundo se han manifestado en contra del uso de los PTC, y han propuesto que deben regirse bajo las mismas políticas regulatorias que se aplican a todos los productos de tabaco, en línea con lo establecido por el Convenio Marco de Control del Tabaco de la OMS.

Palabras clave: Tabaco; Nicotina; fumadores; productos de tabaco; conducta adictiva; daño hepático inducido por químicos y drogas.

\footnotetext{
* Comisión de Tabaco. Sociedad Chilena de Enfermedades Respiratorias.
} 


\section{Introducción}

El consumo de tabaco continúa siendo la principal causa de enfermedad y muerte evitable en todo el mundo, convirtiéndose en una verdadera epidemia durante el siglo XX. Para enfrentarla, la OMS auspició y negoció con los países miembros, el Convenio Marco de Control del Tabaco (CMCT), que se ha considerado como el primer tratado internacional de salud pública, el cual ha sido ratificado en la actualidad por 181 países. Chile lo ratificó y está vigente en nuestro país desde el año 2005.

En los últimos años se observa una tendencia a la disminución del consumo de tabaco, como resultado de las acciones de control del tabaco, la que ha sido dispar en diferentes países. Aquellos países que implementaron políticas integrales de control del tabaco y que lograron superar la interferencia de la industria tabacalera, evidenciaron grandes progresos, con reducciones significativas en el consumo de tabaco ${ }^{1}$. En nuestro país, si comparamos las encuestas nacionales de salud observamos una caída en la prevalencia del consumo de tabaco desde un $43,5 \%$ a un $33 \%$ entre la Encuesta Nacional de Salud del año 2003 a la del 2016-172.

La industria tabacalera tiene una larga historia, desde hace décadas, de promover el consumo de tabaco, introduciendo productos de "riesgo reducido". En los años de 1950 y 60s introdujeron los cigarrillos con filtro, después los cigarrillos light, ultra-light, bajos en alquitrán y una variedad de productos modificados para acallar las crecientes preocupaciones públicas acerca de los peligros de fumar, de tal manera de mantener las ganancias de la industria. En la última década han promovido los cigarrillos electrónicos, genéricamente conocidos como SEAN (Sistemas Electrónicos de Administración de Nicotina) o por su sigla en inglés: ENDS (Electronic Nicotine Delivery Systems).

Actualmente, la industria del tabaco está promoviendo la última generación de los llamados Productos de Tabaco Calentado (PTC), conocidos en la literatura anglosajona como Heat Not Burn tobacco products (HNB). A través de una agresiva publicidad los recomiendan como productos de "bajo riesgo" y promueven su uso diciendo que adoptando estas nuevas tecnologías se pueden salvar muchas vidas ${ }^{3}$. Además en el año 2017 Philip Morris International (PMI) lanzó una Fundación denominada "Por un mundo sin humo" con un presupuesto anual de 80 millones de dólares, durante los próximos 12 años. Esta Fundación tiene como objetivo apoyar la investigación en reducción de daño y "eliminar el humo en todo el mundo"4, a través del uso de estos nuevos productos.

Se examinará a continuación las características de los PTC, los estudios en relación a sus emisiones y sus efectos para la salud.

\section{¿Qué son los productos de tabaco calentado?}

Son dispositivos que utilizan una batería que permite el calentamiento de un "cigarrillo" de tabaco prensado, con sustancias que le dan sabor $\mathrm{y}$ otros químicos, lo que produce un aerosol de nicotina que crea un "golpe de nicotina" similar a un cigarrillo convencional. Estos dispositivos calientan el tabaco a temperaturas entre $300-350^{\circ} \mathrm{C}$, sin quemarlo, ya que la combustión se produce a $800-900{ }^{\circ} \mathrm{C}$, por lo que producirían menos sustancias tóxicas para el fumador.

Es importante aclarar que en la literatura se utilizan diversos términos y que se consideran como sinónimos: Productos de Tabaco Calentado, Sistemas Calentadores de Tabaco, Productos de Riesgo Modificado. En la literatura anglosajona se les llama Heated Tobacco Products (HTP), Tobacco Heat System (THS), Heat-Not-Burn Tobacco (HNBT), Modified Risk Tobacco Products (MRTP).

En el fondo son nuevas y sofisticadas formas para que sus usuarios consuman nicotina en cantidades variables, pero capaces de producir o mantener la adicción a esta droga.

En la actualidad existen tres PTC que se están comercializando internacionalmente: IQOS de la Philip Morris International (PMI), Glo de la British American Tobacco y Ploom TECH de la Japan Tobacco International. Es decir, son las principales transnacionales del comercio mundial de tabaco las que están produciendo y comercializando estos nuevos productos.

Las mayoría de las publicaciones hasta la actualidad se refieren al IQOS, por lo que de aquí en adelante nos referiremos a esta marca, ya que es la que tiene más tiempo en el mercado (desde 2014) y con mayor distribución a través del mundo (actualmente en 38 países).

\section{IQOS}

¿Qué significa IQOS? Es un acrónimo que proviene de la frase, en inglés: I Quit Ordinary Smoking, es decir, yo dejo el tabaco habitual, convencional.

El IQOS tiene tres componentes, necesarios para un funcionamiento adecuado: un soporte que tiene un sistema electrónico que calienta la 
barra de tabaco que se coloca al interior de este, un cargador (que recarga al soporte después de cada uso) y la barra de tabaco. La PMI vende en forma separada los cigarrillos de tabaco prensado o barras de tabaco (Heets o HeatSticks), bajo el nombre de Malboro u otras marcas de cigarrillos. Esto permite que los fabricantes puedan hacer publicidad del IQOS, ya que la legislación que prohíbe dicha publicidad, se aplicaría solo al cigarrillo de tabaco prensado.

El cigarrillo HeatSticks tiene 4 partes: una boquilla-filtro de celulosa, un segundo filtro que es un polímero especial cuya función es enfriar el humo que se produce al calentar el tabaco, después tiene un tubo de acetato y a continuación está el tabaco prensado o compactado.

La Philip Morris International ha invertido más de 6 billones de dólares en la producción y promoción del IQOS y en su página web y en publicaciones propias ha dado a conocer los hallazgos de sus investigaciones. Es importante señalar que la PMI, en diciembre de 2016, presentó una solicitud a la Administración de Alimentos y Drogas de los Estados Unidos (FDA) para que IQOS fuera reconocido como producto de riesgo reducido. Esta solicitud incluyó detalles acerca del producto, la química del aerosol que produce, su toxicología, efectos clínicos, percepciones del producto y su envase. Esta información ha sido analizada por numerosos investigadores, algunas de las cuales se detallan más adelante.

Como resultado de los estudios de la PMI sobre IQOS, la industria dice ${ }^{5}$ :

- IQOS no produce combustión ni humo.

- De acuerdo a test de laboratorio, su aerosol tiene niveles significativamente menores de sustancias dañinas y potencialmente dañinas y es mucho menos tóxico que fumar cigarrillos.

- En un estudio de 90 días en EE.UU. y en otro de igual duración en Japón, reportaron que los fumadores que se cambiaron completamente a los productos de tabaco calentado reducían su exposición a las sustancias dañinas y potencialmente dañinas estudiadas. Estos niveles se aproximaban a los observados en personas que dejaban de fumar durante la duración de estos estudios.

- De acuerdo a test de calidad del aire, el aerosol no impacta negativamente la calidad del aire interior.

- Su investigación muestra que las personas que nunca han fumado o que han dejado de fumar muestran un interés insignificante en el IQOS. Por lo tanto, existe un gran potencial para que los fumadores adultos se cambien de los cigarrillos convencionales a los PTC.
Estas conclusiones de la PMI se deben contrastar con los hallazgos de estudios independientes, que se mostrarán a continuación. En una revisión sistemática de la literatura Simonavicius et al. ${ }^{6}$ estudiaron las publicaciones en relación a los PTC: encontraron 31 estudios evaluados por revisión de pares, de los cuales 20 eran afiliados a la industria del tabaco y solo 11 independientes.

Es importante destacar que las publicaciones actuales se refieren a estudios sobre las emisiones y componentes del aerosol de los PTC y sus efectos agudos o en el corto plazo sobre la salud, ya que por razones obvias de su reciente introducción en los mercados, no hay estudios a largo plazo.

\section{Emisión de sustancias químicas por IQOS}

En un trabajo de Bekki et al. ${ }^{7}$ se midieron las sustancias químicas en el humo de la corriente principal de IQOS y de cigarrillos convencionales. Se compararon cigarrillos normales, tipo 3R4F y 1R5F, con IQOS mentolado y no mentolado, midiendo las concentraciones de Nicotina, Alquitrán, $\mathrm{CO}$ y Nitrosaminas específicas del tabaco (TSNA). En cuanto a nicotina, en cigarrillos normales se encontró 1,7 y $1 \mathrm{mg}$ por cigarrillo, en tanto que las cifras para IQOS son muy similares: 1,1 y 1,2 mg/cig. En cuanto a alquitrán en los cigarrillos normales las cifras son de 25,2 y $19,2 \mathrm{mg} / \mathrm{cig}$ para el IQOS son 9,8 y $13,4 \mathrm{mg} / \mathrm{cig}$, cantidades menores que las de los cigarrillos normales, pero que siguen siendo altas. En cuanto a las TSNA, se encontró que las cantidades de estas nitrosaminas en el IQOS son más bajas que en el cigarrillo normal.

Leigh $^{8}$ comparó los niveles de TSNA en el aerosol de IQOS con cigarrillos electrónicos (MarcTen) y Malboro rojo. Los niveles de TSNA por puff de IQOS fue menor que los cigarrillos convencionales pero mayores que los cigarrillos electrónicos.

Auer el al. ${ }^{9}$ midieron la concentración de hidrocarburos aromáticos policíclicos (HAP) que hay en el humo liberado por el IQOS y lo que contiene la media de las 35 marcas de tabaco más vendidas en EEUU. Las cantidades de HAP en el IQOS es menor que en los cigarrillos normales, fluctuando entre un 1 a un $9 \%$. Pero también encontraron que uno de los HAP, el acenafteno, en los cigarrillos normales se encuentra en concentración de $49 \mathrm{ng}$ por cigarrillo mientras que en los IQOS la concentración aumenta a $145 \mathrm{ng}$. En cuanto a nicotina se encontró $361 \mathrm{ng}$ por cigarrillo normal y $301 \mathrm{ng}$ en IQOS. 
En otra publicación de Davis et al. ${ }^{10}$ se encontró que la utilización de IQOS alcanza temperaturas entre 300 y $350{ }^{\circ} \mathrm{C}$, pero que a esa temperatura se produce pirólisis, lo que permitió la transformación del componente principal del filtro del IQOS, que es un polímero (constituido por epsilon-caprolactona, lactido, 1-2 diacetin y otros compuestos orgánicos desconocidos) en cianidrina de formaldehido, tóxico muy peligroso, incluso fatal en humanos y que se utiliza en la producción de resinas sintéticas y como solvente.

St Helen et al. ${ }^{11}$ revisaron los trabajos presentados por la PMI en su postulación a la FDA para ser considerados como productos de riesgo reducido. Estos autores encontraron que los datos de la PMI mostraban que IQOS redujo la exposición de solo algunas sustancias tóxicas o potencialmente tóxicas (40 de 93) identificadas por la FDA. Además los datos de la PMI mostraron niveles significativamente más altos en IQOS de muchos tóxicos no listados por la FDA, comparado con cigarrillos convencionales: en 22 de ellos los niveles eran sobre el doble y en 7 sobre 10 veces.

\section{Efectos vasculares}

En un artículo de Nabarizadeh et al. ${ }^{12}$ se estudia el riesgo de enfermedad cardiaca por la alteración de la capacidad de las arterias de dilatarse cuando lo requieren para adecuarse a aumentos del flujo sanguíneo (FMD). Este autor evaluó, en un modelo en ratas, la exposición al aerosol de IQOS comparado con el aerosol de un cigarrillo convencional (Malboro Rojo) y aire limpio. El resultado fue que el aerosol de IQOS afecta la función endotelial vascular, medida por FMD, en forma similar a lo que lo hace el cigarrillo convencional.

\section{Efectos pulmonares}

Moazed et al. ${ }^{13}$ encontró que los datos aportados por la PMI en su solicitud a la FDA, ponen una voz de alerta sobre los efectos pulmonares de IQOS. En ratas expuestas a IQOS se encontró inflamación pulmonar e inmunomodulación. En humanos la PMI reporta mediciones de la función pulmonar en fumadores que se cambiaron a IQOS, no encontrando mejoría en ellos.

Leigh et al. ${ }^{14}$ expusieron células del epitelio bronquial humano in vitro al aerosol de tres productos de PMI: IQOS, un cigarrillo electrónico (MarcTen) y un cigarrillo convencional (Malboro rojo), con niveles de nicotina comparables en la interfase aire-líquido. IQOS mostró significativa mayor toxicidad que los cigarrillos electrónicos, pero menos que los cigarrillos convencionales.

Sohal et al en una reciente publicación ${ }^{15}$. evaluaron los efectos de la exposición a IQOS, cigarrillos electrónicos y cigarrillos convencionales en células humanas del epitelio bronquial y musculo liso in vitro. Encontraron que IQOS tiene el potencial de aumentar el estrés oxidativo e inflamación, infecciones, remodelamiento de la vía aérea e iniciar transiciones del epitelio mesenquimal, lo que puede llevar a enfermedades pulmonares crónicas.

\section{Daño hepático}

Es posible que los PTC pudieran causar otros daños que no son causados por cigarrillos convencionales. Shun et al. ${ }^{16}$ analizaron los trabajos presentados por la PMI en su postulación a la FDA para ser considerados como productos de riesgo reducido. Los investigadores de la PMI compararon la toxicidad hepática en ratas expuestas al aerosol de la corriente principal de IQOS comparada con cigarrillos convencionales $\mathrm{y}$ a aire ambiental. Encontraron que algunas medidas de toxicidad hepática (peso del hígado, niveles plasmáticos de alanina aminotransferasa y vacuolización hepatocelular) aumentó más en las ratas hembras (pero no en los machos) expuestas a IQOS que las expuestas al cigarrillo convencional.

En otro estudio de la PMI realizado en humanos $^{16}$ compararon la exposición al IQOS, cigarrillo convencional y abstinencia de fumar, encontrando que hubo aumento de la bilirrubina mayor en IQOS que en los fumadores de cigarrillos convencionales y que los abstinentes.

A la luz de todos estos antecedentes, el Dr. Stanton Glantz, de la Universidad de California ${ }^{17}$ considera que IQOS (y en forma similar otros PTC) son simplemente diferentes que los cigarrillos convencionales y producen menos de algunos tóxicos y más de otros, por lo que IQOS puede producir menos, igual o más riesgos para la salud que los cigarrillos convencionales dependiendo de la patología. También concluye que IQOS emite más toxinas y tiene más efectos adversos para la salud que los cigarrillos electrónicos.

\section{Humo de tabaco ambiental}

PMI ha publicitado que el aerosol de IQOS no impacta negativamente la calidad del aire interior. ¿Qué dicen los trabajos independientes? 
En un estudio de Protano et al. ${ }^{18}$ evaluaron la concentración de Partículas Submicrónicas (un indicador de humo de tabaco ambiental) inhaladas en el pulmón de fumadores pasivos expuestos al aerosol ambiental de cigarrillos electrónicos, IQOS y cigarrillos convencionales. Encontraron que los cigarrillos electrónicos y IQOS generaron 4 veces menos partículas submicrónicas comparado con cigarrillo convencional, pero que una alta proporción de estas partículas son capaces de alcanzar la zona alveolar pulmonar de los no fumadores expuestos, con los riesgos para la salud que ello implica.

Ruprecht et al. ${ }^{19}$ estudiaron las emisiones al aire ambiental interior producidas por cigarrillos convencionales, IQOS y cigarrillos electrónicos. En el análisis del humo ambiental producido por IQOS, estos autores encontraron grandes diferencias en las concentraciones del material particulado dependiendo del tipo de compuestos orgánicos. Mientras los hidrocarburos aromáticos policíclicos fueron casi indetectables, ciertos ácidos orgánicos (como acido suberico, acido azelaico y acidos n-alkanoicos) tanto como levoglucosanos, se encontraron en niveles importantes (hasta 2 a 6 $\mathrm{mg} / \mathrm{h}$ ). Las emisiones de metales fueron menores que los cigarrillos convencionales y que los cigarrillos electrónicos. Otro importante hallazgo fue la presencia de aldehídos carcinogénicos, tales como formaldehido, acetaldehído y acroleína, aunque en niveles menores que los cigarrillos convencionales. Estos autores concluyen que fumar cigarrillos electrónicos y IQOS debe prohibirse en lugares cerrados de uso público.

Cancelada et al. en una reciente publicación ${ }^{20}$ encontraron valores similares a los de Ruprecht en cuanto a acetaldehído, acroleína y menores de formaldehido en el aire ambiental producido por la emisiones de la corriente principal de IQOS. Estos autores encontraron además naftaleno, que no fue reportado por la PMI en su presentación a la FDA. Estos autores concluyen que IQOS contamina en 1 a 2 órdenes de magnitud menores que los cigarrillos convencionales, pero con niveles preocupantes de algunos componentes, especialmente el caso de la acroleína.

\section{Promoción de IQOS como alternativa a los cigarrillos convencionales}

La PMI no promociona el IQOS como alternativa para dejar de fumar, sino que para aquellos fumadores que no quieren dejar de fumar. Para que este predicamento funcione, los fumadores actuales de cigarrillos convencionales deben dejar estos y cambiarse al consumo de IQOS. La realidad muestra otra cosa. Hay estudios ${ }^{21}$ que demuestran que la mayoría de los usuarios de PTC continúan fumando cigarrillos convencionales, es decir se transforman en los así llamados fumadores duales. Incluso, en este mismo estudio, se observó que los usuarios de PTC quedaban como fumadores triples, ya que utilizaban cigarrillos convencionales, cigarrillos electrónicos y PTC. Los efectos de este cóctel son desconocidos. Se puede concluir que la mayoría de las personas usan los SEAN (Sistemas Electrónicos de Administración de Nicotina) y los PTC como complemento a los cigarrillos convencionales, no como una alternativa. Por lo tanto no hay beneficios para la salud de la mayoría de los fumadores e incluso para algunos puede haber un aumento de los riesgos de daño ${ }^{22}$.

\section{IQOS y los jóvenes}

Es necesario considerar lo que está pasando con estos nuevos productos entre los jóvenes y que podría incrementarse más en el futuro. La experiencia con los cigarrillos electrónicos indica que se han convertido en una nueva moda entre los adolescentes de diversos países del mundo, especialmente en los EE.UU. con la marca Juul, lo que ha llevado a las autoridades de salud a tomar medidas de control o claramente a prohibir su venta, como ha ocurrido en varios países y recientemente en San Francisco, California ${ }^{23}$.

La publicidad de IQOS lo presenta como un producto de menor riesgo, con empaquetado atrayente, similar a un IPhone, en tiendas muy elegantes y sofisticadas. Su marketing realza en el producto su limpieza, pureza, el ser chic y una apariencia de alta tecnología, todo lo cual lo hace muy atractivo, especialmente entre los jóvenes ${ }^{24}$.

En un estudio realizado en Italia ${ }^{25}$ casi la mitad de los consumidores de tabaco calentado y más de la mitad de las personas interesadas en utilizar los PTC nunca habían fumado, lo que sugiere que IQOS puede crear nuevas generaciones adictas a la nicotina, además de "renormalizar" el consumo de tabaco en la sociedad.

Es por esto que cuando se evalúan los pro y contras de los PTC y de los SEAN, debe considerarse su impacto en toda la población, no solo en los fumadores, que son una minoría. Desde el punto de vista de la salud pública estos productos pueden tener un efecto neto desfavorable, especialmente por el enganche que pueden producir en los no fumadores ${ }^{22}$.

Finalmente podemos decir que la $\mathrm{OMS}^{26}$ 
considera que todas las formas de tabaco son dañinas, incluidos los PTC. Recomienda entonces que los PTC deben estar sujetos a las mismas políticas regulatorias que se aplican a todos los productos de tabaco, en línea con lo establecido por el Convenio Marco de Control del Tabaco.

Además las principales sociedades de enfermedades respiratorias del mundo han emitido documentos de posición en relación a los SEAN y a los PTC, advirtiendo de los posibles daños a la salud, de que no son alternativas válidas para dejar de fumar y que pueden enganchar nuevos fumadores, especialmente entre los más jóvenes. Las sociedades que han emitido declaraciones al respecto son: declaración conjunta de la Asociación Argentina de Medicina Respiratoria (AAMR), Asociación Latinoamericana del Tórax (ALAT), Sociedad Española de Neumología y Cirugía Torácica (SEPAR), Sociedad Mexicana de Neumología y Cirugía del Tórax (SMNyCT) y Sociedad Chilena de Enfermedades Respiratoria (SER Chile) ${ }^{27}$, Sociedad Europea de Respiratorio (ERS) ${ }^{28}$ y la Unión Internacional contra la Tuberculosis y Enfermedades Pulmonares (The Union) ${ }^{29}$.

Por otra parte, no podemos olvidar que los PTC son manufacturados por la industria del tabaco, la que está interesada en expandir su consumo a la mayor cantidad de personas posible, fumadores y no fumadores. Con estos nuevos productos la industria del tabaco está intentando rehabilitar su reputación, intentando aparecer como parte de la solución y no parte del problema, de tal manera de poder influir sobre los tomadores de decisión.

El juramento Hipocrático establece que los médicos deben defender estándares éticos específicos y con un principio básico: "primero no hacer daño". Los pulmones necesitan respirar aire limpio, no "niveles reducidos de tóxicos y carcinógenos" y el cuerpo humano no está destinado a ser dependiente de drogas adictivas, como la nicotina ${ }^{22}$.

\section{Bibliografías}

1.- U.S. DEPARTMENT OF HEALTH AND HUMAN SERVICES. The Health Consequences of Smoking-50 Years of Progress: A Report of the Surgeon General. 2014. https://www.ncbi.nlm.nih.gov/pubmed/24455788.

2.- https://www.minsal.cl/wp-content/uploads/2017/11/ ENS-2016-17_PRIMEROS-RESULTADOS.pdf (Consultado el 14 de junio de 2019).

3.- PMI SCIENCE. Learn more about the science behind RRPs. https://www.pmiscience.com/welcome (Consultado el 20 de junio 2019).
4. FOUNDATION FOR A SMOKE-FREE WORLD. https://www.smokefreeworld.org/our-vision. (Consultado el 20 de junio de 2019).

5.- https://www.pmi.com/science-and-innovation/ourfindings-to-date (Consultado el 11 de junio de 2019).

6.- SIMONAVICIUS E, MCNEILL A, SHAHAB L, BROSE LS. Heat-not-burn tobacco products: a systematic literature review. Tob Control 2019; 0: 1-13. doi:10.1136/ tobaccocontrol-2018-054419.

7.- $\quad$ BEKKI K, INABE Y, UCHIYAMA S AND KUNUGITA N. Bomparison of chemicals in mainstream smoke in heat-not-burn tobacco and combustion cigarettes. J UOEH 2017; 39: 201-207.

8.- LEIGH NJ, PALUMBO MN, MARINO AM, O'CONNOR RJ, GONIEWICZ ML. Tobacco-specific nitrosamines (TSNA) in heated tobacco product IQOS. Tob Control 2018; 27 (Suppl 1): s37-s38.

9.- AUER R, CONCHA-LOZANO N, JACOT-SADOWSKI I, CORNUZ J, BERTHET A. Heat-Not-Burn (HNB) Tobacco Cigarettes: Smoke by Any Other Name. JAMA Internal Medicine 2017; 177: 1050-1.

10.- DAVIS B, WILLIAMS M, TALBOT P. IQOS: evidence of pyrolysis and release of a toxicant from plastic. Tob Control 2018; 0:1-8. doi: 101136/tobaccocontrol-2017-054104.

11.- ST HELEN G, JACOB III P, NARDONE N, BENOWITZ NL. IQOS: examination of Philip Morris International's claim of reduced exposure. Tob Control 2018; 27 (Suppl1): s30-6.

12.- NABAVIZADEH P, LIU J, HAVEL CM, IBRAHIM 3, DERAKHSHANDEH R, JACOB III P, et al. Vascular endothelial function is impaired byaerosol from a single IQOS HeatStick to the same extent as by cigarette smoke. Tob Control 2018; 27 (Suppl 1): s13-9.

13.- MOAZED F, CHUN L, MATTHAY MA, CALFEE CS, GOTTS J. Assessment of industry data on pulmonary and immunosuppressive effects of IQOS. Tob Control 2018; 27 (Suppl 1): s20-5.

14.- LEIGH NJ, TRAN PL, O'CONNOR RJ, GONIEWICZ ML. Cytotoxic effects of heated tobacco products (HTP) on human bronchial epithelial cells. Tob Control 2018; 27 (Suppl 1): s26-9.

15.- SOHAL SS, EAPEN MS, NAIDU VGM, SHARMA P. IQOS exposure impairs human airway cell homeostasis: direct comparison with traditional cigarette and e-cigarette. ERJ Open Res 2019; 5: 00159-2018 [https:// doi.org/10.1183/23120541.00159-2018].

16.- CHUN L, MOAZED F, MATTHAY M, CALFEE C, GOTTS J. Possible hepatotoxicity of IQOS. Tob Control 2018; 27: s39-s40.

17.- GLANTZ SA. Heated tobacco products: the example of IQOS. Tob Control 2018; 27: s1-s6.

18.- PROTANO C, MANIGRASSO M, AVINO P, SERNIA $\mathrm{S}$, VITALI M. Second hand smoke exposure generated by new electronic devices (IQOS and e-cig) and traditional cigarettes: submicron particle behavior in 
human respiratory system. Ann Ig 2016; 28 (2): 109-12. doi:10.7416/ai.2016.2089.

19.- RUPRECHT AA, DE MARCO C, SAFFARI A, POZZI P, MAZZA R, VERONESE C, et al. Environmental pollution and emission factors of electronic cigarettes, heat-not-burn tobacco products, and conventional cigarettes. Aerosol Science and Technology 2017; 51: 674-84. DOI: 10.1080/02786826.2017.1300231.

20.- CANCELADA L, SLEIMAN M, TANG X, RUSSELL ML, MONTESINOS N, LITTER M, et al. Heated Tobacco Products: Volatile Emissions and Their Predicted Impact on Indoor Air Quality. Environ. Sci Technol 2019. DOI: $10.1021 /$ acs.est.9b02544

21.- KIM J, YU H, LEE S, PAEK YJ. Awareness, experience and prevalence of heated tobacco product, IQOS, among young Korean adults. Tob Control 2018; 27 (Suppl 1): s74-7.

22.- ERS POSITION PAPER ON TOBACCO HARM REDUCTION. Statement prepared by the ERS Tobacco Control Committee. May 2019. https://ers.app.box. com/v/ERSTCC-Harm-Reduction-Position (Consultado el 21 de junio de 2019).

23.- NATIONAL PUBLIC RADIO, Inc (US) https://www. npr.org/sections/health-shots/2019/06/25/735714009/ san-francisco-poised-to-ban-sales-of-e-cigarettes (Consultado el 1 de julio de 2019).
24.- HAIR EC, BENNETT M, SHEEN E, CANTRELL J, BRIGGS J, FENN Z, et al. Examining perceptions about IQOS heated tobacco product: consumer studies in Japan and Switzerland. Tob Control 2018; 27: s703.

25.- LIU X, LUGO A, SPIZZICHINO L, TABUCHI T, PACIFICI R, GALLUS S. Heat-not-burn tobacco products: concerns from the Italian experience. Tob Control 2019; 28: 113-4.

26.- https://www.who.int/tobacco/publications/prod_regulation/htps-marketing-monitoring/en/ (Consultado el 1 de julio de 2019).

27.- ZABERT G, GAGA M, JIMÉNEZ RUIZ C, BULJUBACICH D, GARCÍA G, VÁZQUEZ J, et al. Cigarrillo electrónico y demás ENDS: posición de sociedades científicas respiratorias. Rev Am Med Respirator 2019; 19: $139-45$

28.- ERS position paper on heated tobacco products. https:// www.ersnet.org/the-society/news/ers-position-paper-onheated-tobacco-products (Consultado el 21 de junio de 2019).

29.- THE UNION'S POSITION ON HEATED TOBACCO PRODUCTS (HTP) January 2018. https://www. theunion.org/what-we-do/publications/official/body/ Heated-Tobacco-Product-Union-Position-StatementFinal-25.01.18.pdf (Consultado el 21 de junio de 2019).

Correspondencia a:

Sergio Bello S.

Email: sbelllo.sbs.@gmail.com 